\title{
Brain Computer Interface for Spinal Cord Injury
}

\section{Jinyi Long*}

Center for Brain Computer Interfaces and Brain Information Processing, South China University of Technology, Guangzhou 510640, China

*Corresponding author: Jinyi Long, Center for Brain Computer Interfaces and Brain Information Processing, South China University of Technology, Guangzhou 510640, China, Tel: 412-648-3383; E-mail: aujylong@scut.edu.cn

Rec date: Oct 15, 2014, Acc date: Oct 16, 2014, Pub date: Oct 22, 2014

Copyright: (c) 2014 Long J. This is an open-access article distributed under the terms of the Creative Commons Attribution License, which permits unrestricted use, distribution, and reproduction in any medium, provided the original author and source are credited.

\section{Editorial}

Spinal cord injury (SCI) is a chronic paralysis that needs a longterm health, economic, and social issue worldwide. A major goal of the physicians and therapists is to enhance the restoration of movements lost after SCI. Following decades of research about the central nervous system (CNS) has advanced greatly, however, it is till little known about the inducing successful regeneration, especially in the chronic SCI state [1].

Brain-computer interfaces (BCIs) are used to translate brain activity signals into control signals for external devices. Furthermore, BCI has proven to be a useful tool for providing alternative communication and mobility to patients suffering from nervous system injury [2]. For the patients suffering from SCI, BCI can be an effective tool regarding to improving their quality of life through strengthening the efficacy of the residual neuronal pathways or controlling of replacement devices.

BCI combined with functional electrical stimulation (FES) has been attracted more and more attention for its possibility to restore the basic movements in SCI patients. The BCI technology makes the direct brain control of FES systems possible. BCI-triggered FES system has been build up to help the monkey suffered SCI to control grasping movement, which is not possible after SCI [3]. Furthermore, BCItriggered FES system has also successfully applied to SCI patients. Rohm M [4] designed this system to make an SCI patient to induce movement in the hand, fingers, and elbows with about $70 \%$ accuracy.

Particularly, it would also result in a large gain in quality of life for the SCI patients through the neuroprosthetics to replace their missing or weak movement function. BCIs are often directed at assisting and augmenting the patients' motor functions through brain-controlled robot arms [2], wheelchairs [5], or cursors on computer screens [6]. Furthermore, the BCI system can be used to combined multiple pathways for movements together to make it more viable in everyday applications [7]. A hybrid-BCI system is developed to the control wheelchair of velocity and direction through combining motor imagery and P300 [5]. Furthermore, based on this system, SSVEP and P300 are coupled to improve the asynchronous BCI's ability to distinguish between go and stop commands [8]. Motor imagery based $\mathrm{BCI}$ is also applied to control a robotic-leg orthosis for an SCI patient successfully [2].

Taken together, BCI appears to be promising technology to increase the life quality for the SCI patients through restoring motor functions by affecting neural plasticity or replacing motor functions with computerized devices.

\section{References}

1. Filbin MT (2003) Myelin-associated inhibitors of axonal regeneration in the adult mammalian CNS. Nat Rev Neurosci 4: 703-713.

2. Hargrove LJ, Simon AM, Young AJ, Lipschutz RD, Finucane SB, et al (2013) Robotic leg control with EMG decoding in an amputee with nerve transfers. N Engl J Med 369: 1237-1242.

3. Ethier C, Oby ER, Bauman MJ, Miller LE (2012) Restoration of grasp following paralysis through brain-controlled stimulation of muscles. Nature 485: 368-371.

4. Rohm M, Schneiders M, Müller C, Kreilinger A, Kaiser V, et al. (2013) Hybrid brain-computer interfaces and hybrid neuroprostheses for restoration of upper limb functions in individuals with high-level spinal cord injury. Artif Intell Med 59: 133-142.

5. Long J, Li Y, Wang H, Yu T, Pan J, et al. (2012) A hybrid brain computer interface to control the direction and speed of a simulated or real wheelchair. IEEE Trans Neural Syst Rehabil Eng 20: 720-729.

6. Wolpaw JR, Birbaumer N, McFarland DJ, Pfurtscheller G, Vaughan TM (2002) Brain-computer interfaces for communication and control. Clin Neurophysiol 113: 767-791.

7. Orsborn AL, Moorman HG, Overduin SA, Shanechi MM, Dimitrov DF et al. (2014). Closed-loop decoder adaptation shapes neural plasticity for skillful neuroprosthetic control. Neuron 82: 1380-1393.

8. Li Y, Pan J, Wang F, Yu Z (2013) A hybrid BCI system combining P300 and SSVEP and its application to wheelchair control. IEEE Trans Biomed Eng 60: 3156-3166. 